Voix et Images

voixetimages

\title{
Entretien avec Gilles Archambault
}

\section{Sophie Marcotte et François Ricard}

Volume 31, numéro 2 (92), hiver 2006

Gilles Archambault

URI : https://id.erudit.org/iderudit/012874ar

DOI : https://doi.org/10.7202/012874ar

Aller au sommaire du numéro

Éditeur(s)

Université du Québec à Montréal

ISSN

0318-9201 (imprimé)

1705-933X (numérique)

Découvrir la revue

Citer ce document

Marcotte, S. \& Ricard, F. (2006). Entretien avec Gilles Archambault. Voix et Images, 31(2), 15-22. https://doi.org/10.7202/012874ar d'utilisation que vous pouvez consulter en ligne.

https://apropos.erudit.org/fr/usagers/politique-dutilisation/ 


\title{
ENTRETIEN AVEC GILLES ARCHAMBAULT
}

\author{
$++$ \\ SOPHIE MARCOTTE \\ Université Concordia \\ FRANÇOIS RICARD \\ Université McGill
}

VoIX ET IMAGES Vous avez vous-même réalisé un grand nombre d'entrevues et d'entretiens avec des écrivains d'ici et d'ailleurs. Qu'attendez-vous d'un tel exercice? Qu'est-ce qu'un entretien avec un écrivain peut nous dire que l'œuvre de cet écrivain ne dit pas?

GILLES ARCHAMBAULt Je serais porté à croire qu'il ne faut pas s'attendre à ce que des réponses que nous fournissons à des questions nous apportent quelque éclairage sur les livres que nous écrivons. En revanche, il est tout à fait possible qu'un témoin (lecteur, écouteur) trouve un aspect nouveau dans une œuvre qu'il croit connaître. Est-ce qu'un entretien avec un écrivain peut apporter au lecteur des éléments inédits? Cela est rare, mais possible. Sinon, il faudrait estimer que toute œuvre est parfaite, complète. Or, nous le savons, les livres les plus valables, ceux qui trouvent en nous le plus de résonance, ne sont jamais terminés. Il peut donc arriver que des propos échappés par l'auteur éclairent d'un jour nouveau une partie de son œuvre. VoIX ET IMAGES Quand, comment et pourquoi avez-vous commencé à écrire? Sous l'influence de quels modèles? Avec l'aide de quels «mentors»?

GILLES ARCHAMBaULt Il ne fait aucun doute dans mon esprit que si j'ai commencé à écrire, c'est que je ressentais fortement un manque. L'impression ressentie dès l'enfance que j'étais «étranger». Les modèles sont venus plus tard. Le déclencheur a été la figure d'Honoré de Balzac, lorsque le clergé local a voulu empêcher, vers 1949, qu'on célébrât le centenaire de sa mort.

voIX ET IMAGES Comment vous situiez-vous comme romancier dans le contexte de l'effervescence littéraire des années 1960 au Québec? Quel était votre sentiment à l'égard de l'engagement politique, social ou national des écrivains québécois de ce temps? Quelle était votre "position» ou votre attitude concernant la recherche d'innovation formelle et thématique qui marquait la littérature de ces années?

GILLES ARCHAMBAULT Je n'ai jamais cherché à me situer par rapport au renouveau de la littérature québécoise de ce temps-là. Mes modèles étaient français. Je me suis senti dès le départ un écrivain en marge. L'engagement politique a été graduel et n'a jamais entaché mon écriture. Je ne me suis jamais adressé qu'à un lecteur aux prises avec des démons qui ressemblent aux miens. Quant à l'innovation formelle, je ne 
m’y reconnaissais pas tellement. Ce qui ne m'a pas empêché de m'approprier des techniques narratives, monologue intérieur, découpage cinématographique, etc.

VoIX ET IMAGES Quelle était alors et quelle est aujourd'hui votre vision des rapports de l'écrivain québécois avec la France et la littérature française?

GILLES ARChAMbault J'ai commencé à écrire à une époque où la littérature d'ici n'avait pas encore connu sa véritable naissance. Je lisais donc Balzac, Gustave Flaubert, Stendhal. Je consultais le Figaro littéraire, Les Nouvelles littéraires, mais sans jamais imaginer que j'avais des rapports avec la France, où je n'étais encore jamais allé. Les écrivains français contemporains, Jean-Paul Sartre, Albert Camus, m'étaient familiers. Même si je ne songeais pas à m'inspirer d'eux, il est évident que leur influence a compté. Plus de quarante ans après, je serais porté à croire que seule compte pour un écrivain sa patrie littéraire. Et cette patrie, pour moi, c'est la langue française. Le reste, la production littéraire française d'aujourd'hui, ne m'intéresse pas plus que la québécoise. Seules importent pour moi des œuvres qui me touchent. La plupart du temps, il s'agit d'écrivains dont les histoires de la littérature parlent peu ou pas du tout. Georges Perros, Henry Calet, Louis Calaferte, José Cabanis, par exemple, me sont de constantes sources d'inspiration.

VoIX ET IMAGES Quel rôle la radio et le jazz ont-ils joué dans votre vie intellectuelle et dans votre travail littéraire?

GILLES ARCHAMbaULt Le jazz, que j'ai constamment écouté depuis l'adolescence, n'a que très peu agi sur mon écriture. Certes, il m'est arrivé de chercher à transcrire une joie, une émotion que cette musique m'avait procurées. Jamais toutefois n'ai-je eu recours à un fond sonore pour écrire. Quant à la radio, c'est par le recours à la chronique comme genre littéraire qu'elle a pu influencer mon écriture. Entendez par là : l'obligation de trouver un thème à développer coûte que coûte. J'aime ce carcan. Et pour une raison bien simple. Je ne peux perdre trop de temps alors à me demander le fameux «à quoi bon? » contre lequel je dois me battre constamment quand je me mets à écrire un roman ou une nouvelle. Je deviens alors en quelque sorte un journaliste. Mais un journaliste d'un genre tout particulier. On ne m'impose jamais un sujet, je suis libre d'adopter le ton qui me plaît, d'être triste ou serein, respectueux ou irrévérencieux, selon mon humeur. Je me suis d'ailleurs aperçu que cette licence pouvait être dangereuse. La radio invite souvent à un certain exhibitionnisme, à un certain cabotinage. Toutes choses que la littérature (la bonne) ne permet pas. En somme, la radio m'a fait voir une liberté de ton que mon goût pour l'ascétisme m'oblige à restreindre quand j'entreprends un roman.

VoIX ET IMAGES Si vous aviez à retracer l'ensemble de votre carrière littéraire et artistique, quels en seraient pour vous les grands tournants, les principales étapes? GILles ARChAmbault J'ai peine à considérer mon parcours de vie en terme de carrière. Les livres se sont succédé. Je ne trouve plus à la parution d'un livre la joie qui a été mienne en 1963 lorsqu'on m'a remis mes exemplaires d'Une suprême discrétion. Mais je n'oublie pas que ce qui compte, c'est la permission qu'on m'a donnée de vivre par la littérature une vie parallèle. D'où il ressort que j'aurais moins bien vécu si je n'avais pas publié. Je me demande souvent si j'aurais persévéré à la suite de trop de refus des éditeurs. Cette épreuve, je ne l'ai connue que dans ma jeune vingtaine. Le premier livre publié, j'ai trouvé en moi la force de continuer 
même si pendant de nombreuses années j'ai eu la juste impression de travailler dans l'ombre. C'est un ami éditeur, rencontré vers la fin des années 1970, qui le premier m'a donné l'impression que je n'écrivais pas tout à fait en vain. J'avais trop souvent eu jusque-là la sensation d'être lu par des critiques venus d'une autre planète, qui me paraissaient soit trop provinciaux, soit trop sectaires. Or, grâce à cet éditeur, j'ai eu pour la première fois la certitude d'être lu pour des raisons qui me semblaient convaincantes. En 1981 est venu le prix David. Encore aujourd'hui, plus de vingt ans après, je ne m'explique pas la décision du jury. J'ai accepté avec joie et reconnaissance cette distinction, mais j'aurais souhaité qu'on me prépare à la recevoir. Je m'explique. La plupart de mes romans avaient été reçus distraitement. Jamais de descentes en règle, mais des comptes rendus expéditifs. On reconnaissait que j'avais un ton, mais on m'accusait parfois de me répéter. Tout autour, on sacralisait des romans qui me tombaient des mains. Pourquoi décidait-on soudainement de me consacrer écrivain national? Quelques années plus tard, j'ai reçu un autre prix important, celui du Gouverneur général, pour un recueil de courtes nouvelles, L'obsédante obèse. Curieusement, il m'a semblé que, contrairement au David, ce prix-là arrivait au bon moment, qu'il n'y avait pas maldonne. Ces deux prix sont donc les deux étapes que je peux distinguer dans mon parcours. Pour le reste, je suis à 71 ans aussi hésitant, aussi peu sûr de mes moyens qu'à 30 ans. Quand on me parle de mes livres, il me semble souvent qu'on évoque les romans d'un inconnu. Est-ce bien moi qui... ? Et rapidement, je me demande à quoi tout cela rime. Pourquoi ai-je tenu à publier tant de livres? La seule réponse que j'aie pu trouver est celle-ci: j'ai écrit parce que je n'en pouvais plus de me contenter de vivre. Qu'importent les «étapes » d'une carrière lorsqu'il s'agit de secouer une tristesse profonde et perpétuelle qui a à voir avec la comédie de la vie. Pourquoi m'a-t-on mis au monde?

VoIX ET IMAGES Quand vous revoyez en esprit l'ensemble de votre parcours et de votre œuvre, quelle place vous semble être la vôtre dans le paysage de la littérature québécoise contemporaine? Vous percevez-vous comme un écrivain «marginal»? Quelles affinités vous sentez-vous avec la littérature et les écrivains québécois?

GILLES ARCHAMBAULt Je ne crois pas occuper dans la littérature québécoise une place importante. Il faudrait que je sois complètement aveugle pour penser autrement. Je crois bien que cette constatation ne me chagrine pas tellement. Je crois savoir maintenant que le genre de livres dont je suis capable n'est ni dans l'air du temps ni même proche de la sensibilité de mes contemporains. Mes romans et mes nouvelles ont trop à voir avec l'angoisse de vivre pour être lus massivement. Oui, j'ai conscience d'être marginal. Ce qui ne veut pas dire que j'aurais la force d'écrire pour mes tiroirs. J'ai besoin d'un écho. Cet écho, ce ne sont que rarement les écrivains québécois qui me l'apportent. Je crois fermement à l'existence de la littérature d'ici, je compte des amis parmi ceux qui y participent, mais c'est ailleurs la plupart du temps que je trouve mon inspiration. Au reste, je suis de plus en plus un lecteur de journaux intimes, de correspondances, d'écrits en marge plutôt que de fiction. La plupart du temps, je trouve mon bien chez des écrivains du passé, lointain ou non. Si on me demande si je me sens quand même "écrivain québécois", je réponds d'emblée par l'affirmative. Il serait sot et injuste de prétendre le contraire. Je ne revendiquerais que le droit d'être écrivain à ma (modeste) façon. 
VoIX ET IMAGES Vous avez publié un nombre considérable de romans, de recueils de nouvelles et de récits depuis les années 1960. La critique a souvent qualifié votre œuvre de "discrète", tout en soulignant généralement deux choses: le retour de certains grands thèmes; et la présence, dans chaque livre, d'un même ton, d'une même «voix » reconnaissable et qui vous est propre. Qu'en pensez-vous? Est-ce que ces "permanences» (celle des thèmes comme celle de la voix) sont voulues, ou du moins conscientes? Autrement dit: est-ce que les livres que vous avez déjà écrits influencent de quelque manière ceux que vous écrivez ensuite?

GILLES ARCHAMBAULt Il m'arrive de penser que si on a souvent qualifié mes livres de «discrets», c'est tout bonnement parce que mon premier roman s'intitulait Une suprême discrétion. Il y a aussi que rien dans ma nature ne me prédispose aux éclats, aux déclarations fracassantes. Il en va de même pour mes personnages. Le ton que l'on me reconnaît généralement serait celui d'une certaine intimité. Je serais d'accord dans la mesure où l'on admettrait que ce ton de la confidence n'est pas toujours celui de propos murmurés. La confidence, d'accord, mais aussi, me semble-t-il, un léger désabusement, une ironie. C'est, selon moi, une attitude valable par rapport à la vie. Sur fond de tendresse, une volonté de lucidité. Rien ne m'irrite autant que ce verdict de faiblesse dont on accable mes personnages. Est-ce faiblesse que de constater que nos entreprises sont essentiellement vouées à la mort? Quant à la voix que j'aurais, j'estime qu'elle est indispensable. Autrement, pourquoi écrire? Si on ne vous reconnaît pas à la première page, vous n'êtes pas un écrivain. La dernière partie de votre question me pose problème. Je connais mal mes livres puisque je les abandonne dès qu'ils paraissent. D’où le peu de souci que j'ai des répétitions possibles, des doublons. Une chose est certaine: mes livres ont toujours accompagné ma vie. Sans être autobiographiques, ils sont des témoins très proches de l'homme que j'ai été.

VoIX ET IMAGES Si vous le voulez bien, arrêtons-nous à quelques-unes de ces constantes qui traversent votre œuvre. Quelques thèmes, d'abord, et surtout ceux qui semblent les plus frappants: le temps, le vieillissement, la mort. Comment expliquer cette «hantise»?

Gilles archambault Même enfant, j'étais fasciné par le temps. Adolescent, je ne pouvais entendre certaines chansons sans ressentir au-dedans de moi un trouble profond. Je me souviens par exemple du Retour des saisons de Charles Trenet, des Trois cloches des Compagnons de la chanson. L'émotion qui était mienne à ces moments, je l'ai retrouvée en lisant par exemple Miette de Pierre Bergounioux ou Vies minuscules de Pierre Michon. Fuite irrémédiable du temps, absurdité de notre passage sur terre, etc. Quand Sartre évoquait l'homme, "cette passion inutile», je ne pouvais pas ne pas être d'accord. C'est petit à petit, et à l'approche de ma mort, que je me suis mis à découvrir la beauté du monde. Je n'en ai pas été apaisé pour autant. Car qu'y a-t-il de plus cruel que de s'apercevoir que l'on quittera bientôt tant de beauté? Le vieillissement, je commence à en ressentir la grande misère. Je crois de moins en moins aux vertus de la «sagesse». De la bouillie pour les chats. L'angoisse que j'ai toujours ressentie ne disparaîtra jamais. Même le sommeil, peuplé de cauchemars, ne nous épargne pas. Il est pourtant le meilleur remède contre certaines pensées troublantes.

voIX ET IMAGES Deux autres thèmes reviennent aussi de façon régulière dans vos romans et vos nouvelles: celui de la famille plus ou moins fonctionnelle, et celui du 
personnage de l'homme ordinaire, torturé par la vie et par les responsabilités. En quoi ces thèmes sont-ils liés pour vous aux précédents?

GILLES ARCHAMbAULt N'ayant jamais connu de problèmes matériels, je saurais mal en traiter dans mes livres. Ce qui m'intéresse en revanche, ce sont les responsabilités inhérentes au fait de la vie en commun. Comment peut-on accepter de «fonder» une famille? Comment vivre aisément en se disant qu'on est responsable de la venue sur terre d'êtres humains? Surtout si, comme moi, on a toujours pensé que la vie finit immanquablement par être un jeu sinistre. Je n'ai de réelle sympathie que pour ceux de mes personnages qui assument leurs responsabilités. Pour ceux qui abdiquent, je n'éprouverais qu'une certaine compassion. Plus jeune, c'étaient les personnages marqués par l'échec qui m'attiraient, alcoolos, ratés en tout genre. À 71 ans, persuadé que la réussite n'existe pas, qu'elle est un leurre, je préfère de loin me pencher sur le sort de personnages raisonnablement lucides, visités régulièrement par l'angoisse de vivre, mais trouvant au fond d'eux-mêmes des raisons de continuer. Quant à la famille, quant à la femme avec laquelle vous vivez, ce sont des offrandes que la vie vous a faites. Ne m'intéresse que l'homme qui se demande pourquoi des êtres chers s'agglutinent à lui sans paraître trop en souffrir. Comme le rappelle Georges Perros dans Papiers collés, se surprendre toujours quand, rentrant d'une sortie, on trouve quelqu'un pour nous accueillir.

VoIX ET IMAGES Et l'amour? Beaucoup de vos personnages sont, sous ce rapport, des êtres blessés, parfois même méfiants, plus ou moins désillusionnés dans certains cas. Que peut-on encore dire de l'amour humain dans un roman?

GILLES ARCHAMBaULt Vous avez évidemment raison d'utiliser cet adverbe: pourquoi «encore» parler d'amour? Est-il possible d'innover en traitant de ce thème? Sûrement pas. Ce n'est toutefois pas une raison de l'éviter. La plupart du temps, j'ai choisi dans mes romans et mes nouvelles d'envisager l'amour à l'intérieur du couple. Du couple déjà formé. La révélation des premières rencontres, l'éblouissement initial, ce sont des thèmes qui m'attirent de moins en moins. Tellement les liaisons durables, leurs grandeurs et leurs misères me paraissent un terreau fertile. La simple présence de l'autre, son univers qu'on ne saisit que par bribes, ces certitudes qui deviennent vite des doutes, voilà qui est à mon sens autrement plus mystérieux que l'attirance physique, le désir. Mes personnages masculins ne sont jamais des surhommes. Il arrive aux meilleurs d'entre eux d'estimer que leur compagne est le seul rempart qu'ils possèdent contre l'angoisse. Les femmes que je décris ne sont pas différentes sous ce rapport. Pour que je me penche sur leur sort, mes personnages doivent avoir en eux une faille. À ce chapitre, je serais très proche de Jean Rhys, romancière anglaise que je relis avec assiduité.

VoIX ET IMAGES Vos romans remettent souvent en question les valeurs traditionnelles (famille, mariage, religion), mais sans cynisme ni volonté de contestation bruyante et systématique. Dans quel genre de monde ou de société avez-vous, comme romancier, le sentiment de vivre? Désespérez-vous du monde actuel, ou voyez-vous quelque espoir, quelque possibilité d'y trouver le bonheur?

GILLES ARCHAMBAULt Je mentirais si je prétendais que le vingt et unième siècle dans lequel nous venons d'entrer me paraît hospitalier. Le fanatisme religieux, le culte de l'argent, l'arrogance de l'inculture, comment ne pas les voir? Je dois aussi ajouter 
que je n'ai jamais cru au bonheur dans quelque société que ce soit. Si, par exemple, j'affirme croire en l'indépendance du Québec, cette position n'a rien à voir avec le bonheur, mais plutôt avec un sentiment de la justice. Pour moi, de toute manière, le bonheur ne serait au mieux qu'une absence de malheur. On n'est pas heureux de ne pas avoir mal aux dents, tout juste peut-on se dire alors que, par rapport à la veille, alors qu'un abcès lancinant vous faisait hurler, les choses vont maintenant plutôt bien. VoIX ET IMAGES Pour enchaîner avec la question précédente: sans être un écrivain «social» ou "politique», considérez-vous que le roman, ou du moins que vos romans, ont quelque chose à dire sur la société dans laquelle vous vivez? Ou qu'ils ont, d'une certaine manière, une manière qui pourrait même être oblique ou détournée, une portée politique ou "patriotique» quelconque?

GILLES ARCHAMbauLt J'imagine qu'un sociologue pourrait tirer de la lecture de mes romans une analyse d'une certaine société québécoise. Je ne suis pas sûr toutefois que son analyse m'intéresserait, tellement est ancrée en moi la certitude que si je sens le besoin d'écrire, cela ne dépend ni de ma situation de petit-bourgeois ni des heurs et malheurs de la société dans laquelle je vis.

VoIX ET IMAGES Nous évoquions tout à l'heure cette « voix » dont la critique reconnaît la présence dans votre œuvre. Comment qualifieriez-vous cette voix? Par quels moyens stylistiques tentez-vous de la faire entendre? Autrement dit: quelles seraient les grandes règles ou les grandes exigences que vous vous efforcez de mettre en œuvre dans votre prose? Qu'est-ce, pour vous, qu'une belle prose?

Gilles ARChambault Très tôt dans ma vie, et presque en même temps que je découvrais en moi une sorte de malaise perpétuel, je me suis rendu compte d'une évidence: il fallait que j'écrive le plus clairement et le plus simplement possible des états d'âme qui ressembleraient à ceux qui étaient les miens. Mais clarté ne signifie pas qu'on doive bannir le mystère dans l'écriture. J'aime parfois proposer au lecteur une phrase qui donne d'un personnage ou d'une situation une vision un peu trouble. Comme si le visage ou la scène étaient vus à travers une vitre embuée. Mais la prose est un champ d'exploration infinie. Vous me demandez ce que serait pour moi une belle prose. Je pense en ce moment précis à une phrase d'Émile Cioran dans ses Cahiers: "Je ne prise rien tant qu'une prose squelettique traversée d'un frisson. » Sans le frisson, pourquoi écrire, pourquoi lire?

voIX ET IMAGES Votre dernier roman, De l'autre côté du pont, même si vous y traitez sensiblement les mêmes thèmes que dans vos romans précédents, semble habité par une certaine sagesse, et même par une certaine résignation face à la vieillesse et à la mort. Qu'est-ce qui pourra venir ensuite, si la mort est désormais plus ou moins «acceptée»?

GILleS ARCHAmbault Pour moi, cet autre côté du pont signifie que Louis Audry est tout simplement terrassé par la vie. Il n'espère plus rien depuis longtemps. Ce qu'il demande à Monique, l'amie trop longtemps négligée, c'est la permission d'attendre la mort en sa compagnie. Accepte-t-il la mort? Je ne crois pas. Est-il visité par une certaine sagesse? Je ne crois pas non plus. Il aurait l'impression, en continuant de se révolter, d'être un peu ridicule, voilà tout.

voIX ET IMAGES Vous avez écrit un récit autobiographique, Un après-midi de septembre, consacré à la relation que vous entreteniez avec votre mère. Or, dans le 
reste de votre œuvre de fiction, même dans les tout premiers romans, vous semblez aussi répondre à une certaine forme de "tentation autobiographique», même si ces textes ne sont pas présentés explicitement comme des récits de votre propre vie. Comment expliqueriez-vous la présence presque constante de cette «tentation » dans votre œuvre?

GILLES ARCHAmbaULt L'expression "tentation autobiographique» me convient à merveille. Ce serait une tentation à laquelle on ne cède pas. Encore qu'il me faille ajouter que je n'ai jamais voulu, sauf dans Un après-midi de septembre, comme vous le soulignez vous-mêmes, raconter les péripéties qui ont marqué une vie sans intérêt véritable. Je ne m'intéresse, comme lecteur et comme écrivain, qu'à l'existence intérieure, là où l'on cherche la réponse impossible à une question qui l'est tout autant: pourquoi suis-je sur la terre? Il en va de même pour l'amitié. Je n'ai jamais été curieux que d'une chose en ce domaine : comment l'ami se débrouille-t-il avec l'existence? Le reste, son travail, son compte en banque, ses voyages, rien de tout cela ne m'intéresse vraiment. Quant à Un après-midi de septembre, je ne l'ai écrit que parce que j'étais bouleversé par la mort d'une personne que j'avais aimée, ma mère. C'était sa biographie qui me préoccupait, non la mienne.

VoIX ET IMAGES Jusqu'ici, la critique universitaire s'est tenue plutôt loin de votre œuvre. Quelques articles savants, une thèse de doctorat et quelques chapitres de thèses et de mémoires y ont été consacrés. À quoi cela est-il dû selon vous? Et qu'est-ce que vous pensez de ce que les universitaires écrivent au sujet de votre œuvre?

GILLES ARChAmbault Que quelqu'un s'intéresse à ce que j'écris, cela me paraît toujours étonnant. J'espère ne pas paraître outrecuidant en avouant que je n'ai jamais été troublé par le fait que la critique universitaire se soit tenue, comme vous l'avancez, plutôt loin de mes livres. Je n'aurais pas détesté toutefois qu'un lecteur en qui je me reconnaîtrais et qui serait en même temps un critique reconnu me prouve que je l'ai convaincu et, pourquoi ne pas l'écrire, charmé. Quant à réagir à ce que certains universitaires ont écrit sur mes livres, j'en suis bien incapable, étant donné que je passe ma vie à ne pas lire trop assidûment les commentaires que suscitent mes livres. Il me semble toujours qu'on parle de quelqu'un d'autre. À moins qu'il ne s'agisse d'une descente systématique ou d'une recension trop bête. Dans ces cas bien précis, je me fâche un peu et surtout, je me désespère. En règle générale, je suis plutôt étonné qu'on me lise et qu'on sente de surcroît le désir de commenter sa lecture. Il m'arrive de souhaiter dire «merci». 


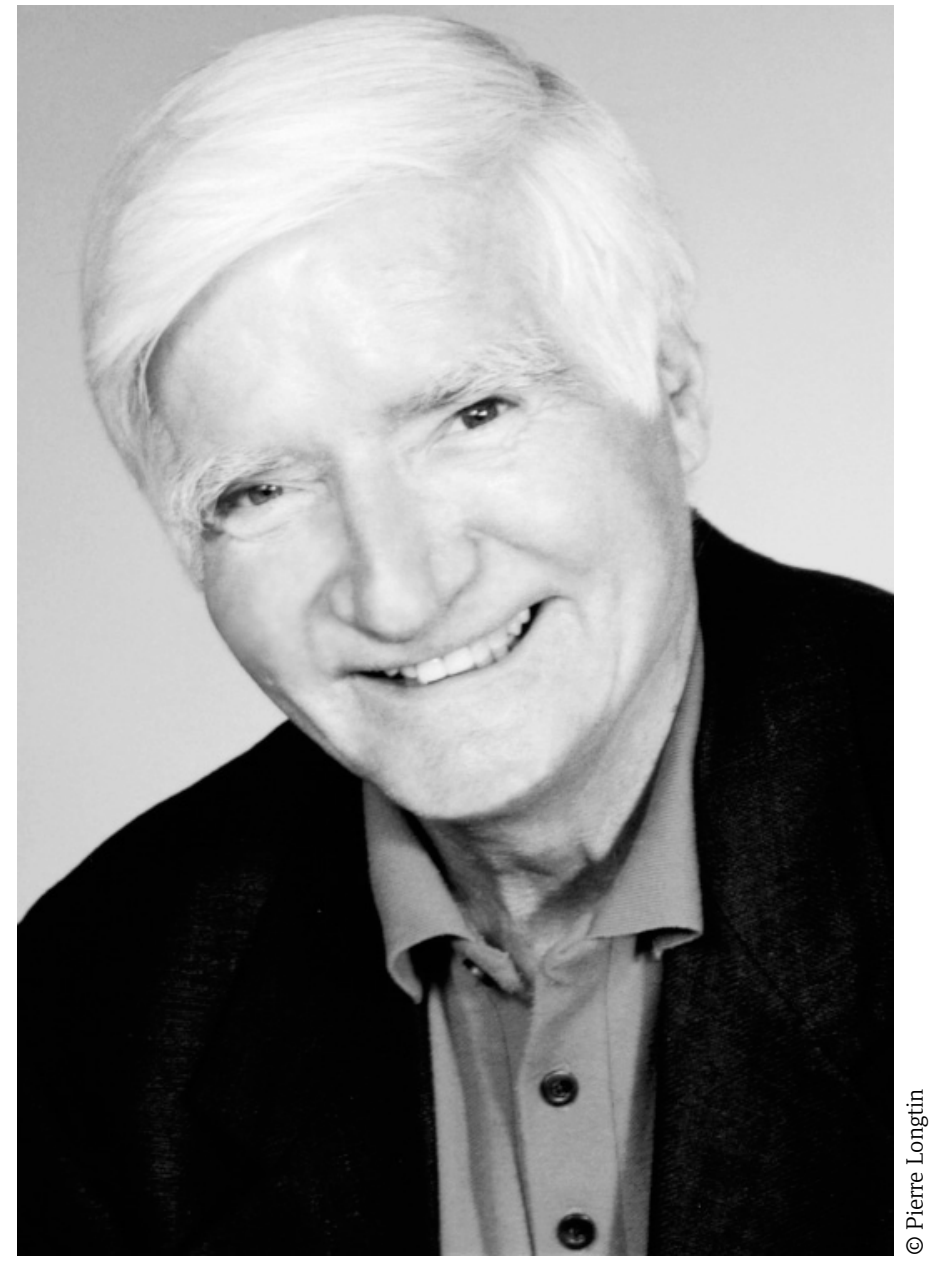

